\title{
Estado actual y tendencias de la investigación en el campo de la construcción
}

\section{sinopsis}

Se presenta a continuación un análisis de las posibles causas que han determinado la situación de crisis en que se encuentra actualmente la inyestigación en España, especialmente en el campo de la construcción, y se exponen algunas ideas orientativas que deberian tenerse en cuenta, a juicio del autor, a la hora de plantear una politica cientific en dicho campo.
El análisis del estado actual de la investigación en el campo de la Construcción, ha de hacerse considerando que toda construcción se realiza para satisfacer un deseo humano. La raíz de este deseo primario se adentra en la propia naturaleza del hombre y de su relación con el medio que le rodea. Por lo tanto, la investigación en el campo de la construcción tiene que estar subordinada: por una parte, al conocimiento de cuáles son los deseos humanos, y por otra, a buscar los caminos para facilitar los medios con los que el ser humano pueda satisfacerlos.

El análisis de los deseos humanos precisa unos estudios sistemáticos que han de fundamentarse en la influencia de la climatología, características geográficas, costumbres, nivel económico, etc. propios de fisiólogos, sicólogos, sociológos, ecólogos, economistas; sin olvidar el aspecto artístico. Partiendo de los resultados de estos estudios el investigador, en la problemática de la construcción, habría de orientar y planificar su labor investigadora.

Por supuesto que no es suficiente con señalar los deseos humanos, sino que éstos hay que confrontarlos con las realidades económicas y técnicas del país. De esta confrontación nacen los niveles de bienestar, que son las aspiraciones que el hombre siente y es necesario ofrecerle por ser etapas ya conseguidas en el progreso continuo de la humanidad.

Este progreso del hombre se inició con su aparición en la Tierra como tal, ante la necesidad de protegerse del medio hostil que le rodeaba, por lo que de un modo inconsciente para él fueron evolucionando sus exigencias humanas. Exigencias que, en un principio, se limitaban a la seguridad; esto es, a su protección contra los animales salvajes, las temperaturas extremas, precipitaciones, vientos y otros fenómenos que él no podia controlar.

\author{
R. MUÑOZ MARTIALAY, \\ Dr. en Ciencias Físicas
}

Es muy reciente el momento en que el hombre se ha sentido seguro y ha tomado consciencia de su situación en la Tierra, lo que le ha creado nuevas exigencias, como son: las fisiológicas, sicológicas, sociológicas y económicas, entre otras.

Este desfase entre las necesidades primarias de supervivencia del hombre, que le ha ido condicionando a lo largo de su existencia, con las posibilidades que le va proporcionando el conocimiento de las ciencias, hace que los estudiosos en el campo de la construcción no dispongan de datos concretos sobre cómo orientar la investigación para conseguir ofrecer al hombre lo que precisa.

Esta falta de datos concretos, junto con las tendencias actuales de fundamentar los fondos destinados a la investigación -en su posible rentabilidad - crea una cierta incertidumbre sobre cuáles han de ser las direcciones que debe seguir la investigación en el campo de la construcción.

Hay científicos que creen se debe dar preferencia al estudio de los nuevos materiales, ya que los tradicionales son suficientemente conocidos $y$, por consiguiente, no deben dedicarse a su estudio más esfuerzos que los que se derivan de su propia aplicación. 
Otros, por el contrario, opinan que los materiales tradicionales pueden ofrecer posibilidades desconocidas aún, por no haber sido suficiente y sistemáticamente estudiados.

Estas dos tendencias plantean dos aspectos fundamentales de la investigación: a largo y a corto plazo. La búsqueda de materiales nuevos y el conocimiento de su comportamiento en el transcurso del tiempo no permite dar soluciones rápidas a un problema concreto con garantía suficiente. Es más fácil, normalmente, cuando se plantea un determinado problema recurrir a su solución extrapolando los resultados ya $\mathrm{co}^{-}$ nocidos o bien estudiando el comportamiento de un determinado material ante el fenómeno en cuestión sabiendo por experiencia que no presenta contraindicaciones frente a otros fenómenos a los que puede estar igualmente sometido.

Es decir, que ante un problema especifico de aislamiento térmico en unas condiciones especiales, si ya se conoce el comportamiento de un material frente a cambios bruscos de temperatura, humedad, esfuerzos, presiones, etc., el estudio de su posible aplicación resultará más rápido y económico y los resultados ofrecerán mayor garantía que si se busca un nuevo material.

Ahora bien, limitarse al empleo únicamente de materiales tradicionales supone perder muchas de las posibilidades que da la naturaleza y que se van descubriendo a medida que avanzan los conocimientos en las distintas áreas de las ciencias.

Por todo lo expuesto, en el campo de la Física de la Construcción, la situación actual puede analizarse bajo los tres siguientes aspectos:

- Conocimiento de las exigencias humanas.

- Investigación en técnicas y materiales tradicionales.

- Búsqueda y aplicación de nuevos materiales.

El primero de estos tres aspectos se escapa de la temática de la Física de la Construcción. No puede incluirse la evolución de las exigencias humanas ya que su estudio corresponde a los fisiólogos, desde el punto de vista biolóqico del hombre; a los sicólogos, dada la capacidad pensante del ser humano; a los sociólogos y ecólogos, en función de las costumbres, posibilidades económicas y técnicas, y características geográficas del medio; así como a otros parámetros, como los artísticos. Conviene no olvidar que la arquitectura es una de las bellas artes y con la que el hombre está más vinculado en la actualidad, pues cada día se encuentra más encerrado por los modelos arquitectónicos y con menos tiempo para dedicarse a las demás artes.
Para analizar la situación actual de los otros dos aspectos, en los que quedaría limitado el campo de actuación de la Física de la Construcción, conviene tener en cuenta que el planteamiento de su investigación es distinto al que se suele hacer en otras ramas, como es en la química, medicina o telecomunicación.

En la construcción desde que un edificio u obra civil se imagina, hasta su terminación, pasan varios años, y un espacio de tiempo tan dilatado no favorece a la decisión de aplicar un nuevo procedimiento o emplear un nuevo material, ante la serie de riesgos de tipo económico y de prestigio que ello implica.

Ante esta situación resulta preciso dedicar atención a todo lo que fomente una mayor conexión y apoyo mutuo entre el Instituto Eduardo Torroja y los sectores demandantes, tanto de la industria privada como del Estado. Resulta claro que el interés de la industria ha de ser mayor por la investigación de aplicación técnica y de desarrollo, con programas que puedan dar resultados a corto y medio plazo, que por la investigación de base. La investigación científica y la investigación pura, al no interesar al sector privado por sí mismas, parece lógico ubicarlas, en todo cuanto sea viable, en la vertiente del Estado.

Las industrias más importantes con que cuentan los paises suelen estar controladas por un número muy limitado de personas. Como puede ser la industria naval, la de productos petroquímicos, siderurgias, etc. Sin embargo, la industria de la construcción se extiende prácticamente a todo el país puesto que se encuentra en todas partes, ya que condiciona la forma de vivir de sus habitantes.

Por ello la industria de la construcción tiene que representar, y de hecho representa, un porcentaje muy importante del P. N. B. (producto nacional bruto). Efectivamente, en edificación se gastan del orden de 230.000 millones de pesetas al año, y en obra civil la cifra es de unos 170.000 millones. Estos 400.000 millones de pesetas al año supone casi un $11 \%$ del P. N. B.

Estas cifras tan importantes se hallan repartidas en numerosas empresas, hasta el punto de que sólo el $40 \%$ (160.000 millones de pesetas) corresponden a entidades con más de 250 productores, y el $80 \%$ del número total de empresas cuenta con menos de 10 productores. 
Una industria tan fragmentada influye muy negativamente cuando se piensa en programas de investigación a largo plazo.

Por otra parte, el Estado tiene prácticamente abandonada la investigación. Basta recordar las conclusiones de la Asamblea de Personal del Instituto Eduardo Torroja, en noviembre de 1974, y que fueron recogidas, a su vez, por el presidente del Consejo Técnico-Administrativo en el discurso de apertura de la 2. ${ }^{a}$ Asamblea de dicho Instituto, que se celebró a continuación y que dijo: «El personal del Instituto cuyas ideas coinciden con las nuestras, cree que a la Administración, que no es más que el reflejo de los españoles, no le interesa la investigación." Por ello no vale la pena insistir una vez más "sobre el ridículo 0,2 \% que el Estado dedica a la investigación; pero eso sí, los buenos deseos y el gran futuro que tiene por haber tomado conciencia el país de la importancia de la investigación...».

Todo lo dicho se refleja en la situación caótica de la investigación en España, en general, y de la construcción en especial.

Pero no cabe duda que un buen replanteamiento de la problemática de la construcción, conducirá a un aumento en los fondos destinados a la investigación en ese campo, ya que con una aportación del $1 \%$, cifra mínima a la que se aspira en los planes de desarrollo, de los 400.000 millones de pesetas, se dispondría de 4.000 millones y esta cantidad resulta casi el doble de lo que el pais dedica a todo el CSIC. Por consiguiente no debería ser el problema económico un obstáculo decisivo en la investigación de la construcción.

Otro aspecto a tener en cuenta es lo poco desarrollada que está la construcción en relación a otros campos: como el de la medicina, o los transportes. Las técnicas que se emplean actualmente en la construcción, y especialmente en la edificación, apenas han variado en los últimos 50 años, y se puede predecir que dentro de los próximos 50 años los edificios serán casi como los actuales en lo que se refiere a su función fundamental: hacer habitable una parte del espacio. Esta evolución lenta de la construcción es inevitable, pues tanto las obras civiles como los edificios hay que plantearlos para que duren muchos años, lo que impide un cambio brusco en la mentalidad de los hombres, como seria preciso que se produjese para adaptarse, por ejemplo, a vivir en una casa-burbuja de plástico.

Este necesario cambio de mentalidad también habría de darse para que el hombre se conformara con vivir en ciudades construidas por sistemas industrializados que impiden crear una variedad suficiente para darle la posibilidad de elegir su propio modo de vivir.
- Existe la posibilidad de que la investigación en el campo de la construcción tenga una ayuda económica relativamente suficiente.

- Es admisible un planteamiento de la construcción, sin incluir novedades que sólo sirven de especulación a ideas futuristas.

Por consiguiente, si el módulo de los edificios actuales no ha de sufrir una transformación fundamental, la investigación habrá de orientarse hacia los materiales básicos: piedra, madera, cemento, acero, vidrio y, también, plástico; y hacia los servicios: iluminación, ventilación, insonorización, higrotermia, agua, gas, telecomunicación, etc

La investigación sobre los materiales básicos, por si mismos, se puede decir que al menos en sus propiedades más significativas, y no entrando en una investigación básica, son suficientemente conocidos. Pero en cambio hay un gran campo de actuación en su aplicación, en lo que se refiere a economía. Esto es, un cerramiento de fachada de obra de fábrica no presenta problemas en cuanto a sus características estructurales y de durabilidad, dada la gran experiencia que existe; pero no es asi respecto a su comportamiento como aislante térmico y acústico. Especialmente en lo que se refiere al planteamiento económico como, por ejemplo, en el sentido de qué es más conveniente: mejorar el aislamiento térmico mediante la ejecución de una cámara de aire, o bien aumentar la potencia de la instalación de calefacción de modo que contrarreste el menor aislamiento.

Esta línea de actuación es muy interesante. La tendencia de fijar unos módulos de construcción es real, pero no lo es tanto saber el nivel de calidad que se puede conseguir dentro de los límites de cada módulo.

Convendría establecer unas condiciones minimas exigibles para cada módulo y tratar de estudiar la aplicación de los materiales para conseguirlos. Es decir, que siguiendo con el ejemplo del cerramiento, de fachada, si se fija un coeficiente de aislamiento térmico de aire a aire de $1,3 \mathrm{kcal} \cdot \mathrm{m}^{-2} \cdot \mathrm{h}^{-1} \cdot{ }^{\circ} \mathrm{C}^{-1}$ y un precio limite, la investigación consistiria en estudiar la eleccion de los materiales y todas las posibles combinaciones para cumplir las condiciones previas: relación de superficie ciega a superficie acristalada; combinación de distintos materiales estableciendo o no cámara de aire; evitación de puentes térmicos, aportaciones de energía; etc.

Tampoco se puede olvidar el campo de la construcción industrializada. Guste o no, hay que recurrir a ella. Con los sistemas tradicionales de construcción son necesarias más de 25 horas de mano de obra por $\mathrm{m}^{2}$ construido. La necesidad creciente de viviendas por una parte y la escasez y coste de mano de obra por otra, obliga a recurrir cada vez más a la industria. 
En la construcción industrializada la investigación puede orientarse en dos aspectos: el humano y el material. El primero se refiere a la búsqueda de soluciones, dado el carácter individualista del español, que resuelvan el problema de la monotonía y el de la sensación de sentirse disminuido ante la presencia de enormes masas uniformes de edificios desprovistos de cualquier elemento singular.

El segundo es el que debe resolver los problemas de control de costes, estudios de movimientos, dimensionado de módulos, características de paneles, etc.

La aplicación de sistemas poco experimentados ha conducido a algunos fracasos, lógicos en toda innovación, pero que justifican to dicho anteriormente sobre la dificultad de aplicar nuevos materiales y procedimientos ante la posibilidad de que aparezcan problemas que en un principio no se habian previsto. Un caso frecuente ha sido la aparición de humedades en el interior de las viviendas construidas a base de paneles de hormigón.

Otro factor a tener en cuenta en la construcción industrializada es que ésta precisa una ejecución muy cuidada para que todos los elementos encajen bien. Es necesario mentalizar a los operarios en la problemática de las tolerancias y de que ya no pueden usar el metro de albañil. El albañil tiene que dejar de ser un obrero manual para convertirse en un mecánico especializado.

Este es un punto muy importante ya que entra de lleno en un campo cuya existencia se ignoraba en la construcción y que, actualmente, se olvida con mucha frecuencia, que es el de la calidad. Ha habido muchos profesionales, y aún quedan algunos, que no creen en la industrialización porque suponen que han de pasar muchos años para llegar a desterrar a los tantos "chapuceros" metidos en la construcción.
La investigación con vistas a la industrialización debe forzarse para evitar que se construya con materiales y sistemas faltos de calidad. Es imprescindible que los centros de investigación estudien y analicen las caracteristicas de los nuevos materiales y métodos constructivos.

En este sentido el Instituto Eduardo Torroja, a través del Documento de Idoneidad Técnica, puede prestar un servicio incalculable; pero, para ello, es necesario disponer de un potencial humano con un equipamiento del que, en la acutalidad, no dispone.

La limitación de recursos del Instituto obliga que los Documentos sólo condicionen el cumplimiento de ciertos requisitos y normas técnicas, y a contar con la experiencia de construcciones ya terminadas.

El que la investigación fuera en vanguardia de la industria podria repercutir muy favorablemente en el desarrollo de las nuevas técnicas. Una ayuda a la investigación por parte de la industria haría disminuir el desfase actual de España con Europa, daría una cierta independencia de la técnica y una posibilidad de exportación, en lugar de importar procedimientos constructivos.

Ahora bien, con construcción tradicional o con construcción industrializada, los edificios, en lo que se refiere a su seguridad, están bastante bien resueltos. Es natural que lo primero que se debe exigir a toda obra es que resista, y en este sentido la investigación tiene ya poco que hacer. La realidad es que los edificios no se caen; la prueba es que cuando alguna vez sucede se produce una gran conmoción en el pais y se recuerda y se habla del suceso durante meses y años. Pero, además, la causa es rara vez por un fallo en los cálculos o por una mala elección de los tipos de materiales; lo corriente es que suele ser debido a una deficiente ejecución o al empleo de materiales en malas condiciones y, en cualquier caso, nunca debido a falta de conocimientos.

La investigación en el sentido de la seguridad tiene su interés en edificios singulares, en edificios de altura y, también, cuando se trata de estudiar un nuevo sistema de prefabricaciór.

El usuario en el momento que entra a ocupar una vivienda no siente la menor preocupación por la seguridad del edificio. No se da el caso de que pida los planos o baje a los scitanos para comprobar la cimentación, y no sólo porque no sepa, simplemente porque no se le ocurre, pues siempre podría recurrir a un técnico.

No sucede lo mismo con los servicios. los cuales son utilizados conscientemente por el hombre y representan el confort; sabe que la ciencia y la industria están en un progreso continuo y exige cada día nuevos servicios y mayor calidad. 
Los servicios ocupan un campo fundamental en la investigación, pues esas mayores exigencias del hombre se traducen en un aumento de la contaminación ambiental, en una escasez de agua y en una disminución alarmante en la disponibilidad de energía.

Ahorrar energía es una necesidad prioritaria de la sociedad y la construcción; con su conservación y entretenimiento consume un $70 \%$ de la energia total que se gasta en el país.'

Actualmente una vivienda corriente para cinco personas consume del orden de 15 millones de calorías al año, lo que representa un coste de unas 70.000 pesetas.

Si estas 70.000 pesetas se multiplican por los 7 millones de familias con que cuenta España, no cabe duda que la cifra que se obtiene es suficientemente justificativa para acometer una investigación en muchos aspectos, incluso en el problema del transporte de esta energía hasta los propios puntos de consumo.

Este simple planteamiento ha llevado a la conclusión de que para viviendas aisladas en las montañas es económicamente más rentable recurrir a pequeñas unidades de potencia que transportarla a través de un tendido eléctrico de varios kilómetros, aparte de la seguridad de servicio y teniendo en cuenta que el entretenimiento que precisan es mínimo.

El aumento constante de los consumos de energía de las grandes ciudades obligan cada vez más a poder disponer de instalaciones de control y regulación asequibles al mayor número de viviendas.

Las grandes ciudades plantean muchos problemas y entre ellos el del ruido es uno de los más preocupantes. Si el problema del ruido se representara gráficamente resultaría una curva exponencial, como lo es la escala decibélica. El aislamiento acústico de un edificio está resuelto teóricamente, pues siempre se puede construir un cerramiento suficientemente aislante, pero los cerramientos han de llevar ventanas y puertas por las que pasan fácilmente los ruidos exteriores. Lo mismo que en un aislamiento térmico se producen puentes térmicos; en acústica existen puentes acústicos, a través de las puertas y también de las grietas que, en ocasiones, aparecen en los paramentos.

La investigación de las condiciones ambientales $y$ de las instalaciones de los edificios necesita un replanteamiento urgente a corto y medio plazo y con una base cientifica seria, asi como dedicar unos fondos suficientes para su financiación.

Pero conviene insistir en que todo lo expuesto no significa que sea necesario destinar unos recursos excesivamente fuertes para la investigación en la construcción, como pueden ser los que se destinan a la defensa del país. Sería, posiblemente, suficiente con un encauzamiento racional de los actuales presupuestos y una aportación de los sectores interesados.

Convendría fomentar ciertos campos de investigación, pero sin olvidar los cementos, hormigones, ladrillos y otros materiales clásicos que aún pueden dar mucho de sí. Lo importante es que una vez decidida una línea se mantenga con continuidad, sin esperar éxitos de gran transcendencia e inmediatos. Estos éxitos son frutos más bien de un azar afortunado, por supuesto no criticable ni despreciable, pero ajenos a la investigación.

Tampoco hay que tomar como válidas frases tales como: "Con la ciencia se puede hacer todo". La realidad es que los científicos investigan y consiguen éxitos que no suelen ser suficientemente satisfactorios al hombre ya que por importantes que sean los avances nunca resolverán todos los problemas que éste tiene; aunque es justo reconocer que las muchas ventajas que posee hoy la humanidad se las debe, en gran parte, a la investigación.
Etat actuel et tendences de la $\mathrm{Re}$ cherche dans le domaine de la Construction

R. Muñoz Martialay, docteur en Sciences Physiques

Ici est présentée une analyse des causes possibles qui ont provoque la situation de crise où se trouve actuellement ialement dans le domaine de la Cons truciion. L'auteur expose quelques idees d'orientation qui devraient, à son avis être tenues en compte au moment de projeter une politique scientifique dans ce domaine.
Current State and trends of Research in the field of Construction

R. Muñoz Martialay, doctor in Physical Sciences

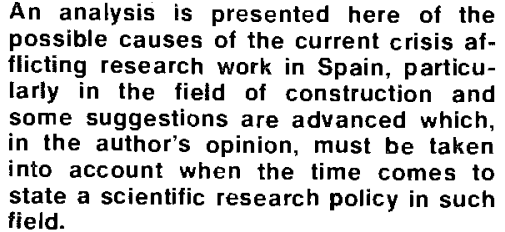

An analysis is presented here of the possible causes of the current crisis atflicting research work in Spain, particusome suge fordion and in the author's opinion must be taken account when the time comes to field.

\section{Derzeitiger Stand und Tendenzen der Forschung Im Bauwesen}

R. Muñoz Martialay, doctor der Pnysik

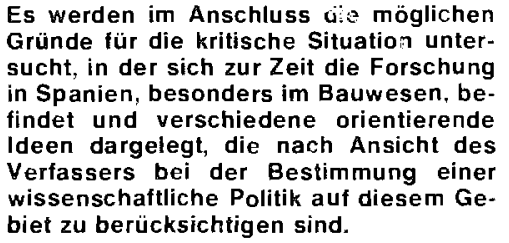

werden im Anschluss o: möglichen Grüde tür die kritische Situation untersucht, in der sich zur Zeit die Forschung in Spanien, besonders im Bauwesen, beindet und verschiedene orientierende Verfassers bei der Bestimmung einer wissenschaftliche Politik auf diesem Gebiet zu berücksichtigen sind. 